\title{
Opinion \\ Nanotechnology and environmental law: the principle of precaution and prevention
}

Volume 3 Issue I - 2016

\section{Opinion}

The Constitution of The Federative Republic of Brazil 1988, article 225 , brings the right we have to an ecologically balanced environment, and their essentiality to the healthiness of the quality of life. Raises him as good of common use - diffuse right - and is responsible for all the duty to defend it and preserve it. To affect these assurances, the Government has the duty - among others - to require prior environmental impact study and control the production, trade, techniques, methods and substances that bring risk to the ecosphere. And here, they do not exclude the engineered for (NanoTechnoScience)

Nanotechnology forges and manipulates atoms and molecules in the 10-9 greatness factor meter. The goal is to build new materials and structures, increasing efficiency and reducing the raw material used in the production process. The promise is to supplant human ills, eradicate hunger, disease and other pressing needs. We entered the Nano Age. A new industrial revolution comes into play the interdisciplinary 'nanoalchemists'.

The subject touches the unreal and fictional, but already find on the shelf several nanoproducts From shampoos, clothes, computer parts, cosmetics, condoms, water filters, medical devices, food coatings, dyes, paints, waterproofing, pesticides, in short, the list is huge and possible areas too - no wonder the nanopatents are increasing.

Faced with all these potential benefits of nanotechnology, one should emphasize the complexity of interrelationships that exist in the ecosystem and the occurrence of nanorisks

To be summarized in one billionth of meter scale, there are the so-called emerging phenomenon, whose biophysical and chemical intersections become more complex. What is presented in the macro scale reveals other properties in the nano. Greatness is decreasing find more instability, new forces and effects; the laws of quantum mechanics appear; the toxicity of a component is enhanced due to the increased contact area ; barriers, protective prior become permeable. In an extreme view, there are nano terrorists opposed to technology, stating that self-replicating nanoparticles destroy the Earth the Gray goo would not be impossible occurrence.

No longer are few studies pointing out the risks posed by nanoparticles that penetrate the basal layer of the skin (nanocosmetics), as well as its storage in soil, water (nanopesticide), air (nanodust, nanopollution), which would result in the degradation and pollution environmental.

At the other extreme, thanks to nanotechnology developments, major advances have occurred in several areas. And that creates a promising and vibrant expectation for such technologies quickly reach all and benefit humanity. In this scenario, it is necessary to consider whether these challenges of this nanorevolution will be protected under national environmental law, or whether such a development will achieve sustainability.

The precautionary principle has been coined as a reserve to abstract dangers of this risk society. Links to situations where it is

\author{
Andre Luiz Aguiar \\ Consultant and Researcher in Nanotechnology, AC\&P-Nano, \\ Brazil
}

Correspondence: Andre Luiz Aguiar, Lawyer, Consultant and Researcher in Nanotechnology - AC\&P-Nano, Brazil, Tel 4I8443-8430Email andre.ot12@gmail.com

Received: January II, 2016 | Published: January 20, 2016

unaware of the potential harmful nanoimpact of or nanoactivities products. In nanotechnology harvest, motivate, at its most extreme, almost a moratorium on research and development. However, is not what drives the Brazilian Constitution, as it wants national progress, as well as environmental protection, founded on human dignity and the ecological balance?

The question arises whether that principle cover what has already been developed, since there are several commercially available nanoproducts. They are in almost all productive chains, which eventually will lead to the existence of nano rubbish dumps and nanolandfill.

Studies indicate possible specific dangers of nanoparticles. As damage to DNA the effects of asbestos, autoimmune diseases. Faced with these findings, caution would not meet the demands of those potential activities and effectively polluting already circulating in the market. Thus, the precautionary principle would fit more in this area, especially for being in many environmental standards bias. What would trigger the legal requirements for impact assessments, licensing and potentially polluting activities review.

Precaution and prevention found in national environmental laws can serve as a footstool in our times. But specific regulatory frameworks of nanotechnology would be one of the challenges that environmental legislation cannot forget. But it does not create an unnecessary delay for technological research and development that the country needs and should be engendering. Social and critical engagements for sustainable directions of nanotechnology are necessary actions to preserve the environment.

The development calls for passage, however, the environmental protection and conservation complain tutelage. On the one hand, there are constitutional principles for the promotion of scientific development, on the other, the duty of all to preserve the biosphere.

\section{Acknowledgments}

None.

\section{Conflicts of interest}

None. 